\title{
EXPANDING ENVELOPES OF STARS
}

\author{
A. A. BOYARCHUK* \\ Krymskaya Astrofizicheskaya Observatoria, U.S.S.R.
}

\begin{abstract}
Observations of Be stars are summarized and discussed, with a view to establishing dimensions of, and physical conditions within, their envelopes. Shell stars and Be stars are considered together, the observed differences between them being considered as due to the size of the envelope and the angle that the rotational axis makes with the line of sight. Rotational ejection of matter is insufficient to account for the formation of the envelopes. Theories of the structure of envelopes are also discussed. The envelopes of Wolf-Rayet stars are considered in a similar way. The interpretation of these is complicated by the known binary nature of many Wolf-Rayet stars. Other groups of stars possessing envelope-like structures - U Gem stars and old novae, symbiotic stars, and T Tauri stars are briefly mentioned. The problems of stellar envelopes, first listed by Struve thirty years ago, are still unsolved.
\end{abstract}

\section{Introduction}

A rather large number of stars have in their spectra some characteristics which indicate the existence of a great mass of gas above their photospheres. In general, in these cases, one says that the stars have extended atmospheres or envelopes,

The distinction between an envelope of a star and an extended atmosphere of a star is somewhat indefinite. Often, some astronomers consider a particular star to have an envelope while other astronomers consider the same star to have an extended atmosphere. One can consider from a general point of view that the density of the gas in envelopes decreases outwards slower than that in extended atmospheres. The density in an envelope can even begin to increase a little at a certain distance from the stellar photosphere. Unfortunately, not many investigations of the density of a gas in stellar envelopes have been made. Therefore, in order to define to which group a given star is related we will make this distinction: if in a stellar spectrum different groups of lines characterize different densities of gas (broad hydrogen lines with strong Stark wings and narrow deep cores; rotationally broadened helium lines and very narrow ionized metallic lines) then we will consider that this star has an envelope. On the contrary, if the spectral characteristics give evidence that the conditions in the outer levels of the atmosphere change smoothly, then we will say that this star has an extended atmosphere.

Thirty years ago, Otto Struve (1942) who gave very much attention to investigations of envelope stars, published a well-known review of problems of gas envelopes in which he formulated the basic problems:

(1) Why do some stars possess tenuous outer atmospheres or shells, while other stars, apparently of identical physical characteristics, do not have such shells?

(2) What is the origin of a shell and how is it supported, in apparent violation of the laws of mechanics?

* Presented at Parksville by J. Smak. 
(3) How can we account for the remarkable tendency of nearly all shells to vary either periodically or, more often, in an irregular manner?

(4) Why do some shells expand, while others are stationary?

In spite of many papers on stellar envelopes being published during the last thirty years, the problems mentioned above have not been solved. Now we will give a brief review of our knowledge on envelopes of stars. We will begin with Be stars, then we will consider other types of envelopes.

\section{Be Stars}

These stars are the most typical representatives of stars having an envelope. The existence of rather narrow emission lines belonging to hydrogen, to helium, and, more rarely, to ionized metals, in addition to the normal B-type spectra indicate the existence of an envelope. Besides the Be stars, there are the stars whose spectra have narrow absorption lines of hydrogen, helium, and once-ionized metals. According to a suggestion of Struve, such stars are called now 'shell-stars'.

Figures 1-3 show the line profiles observed in spectra of different Be stars. Normal

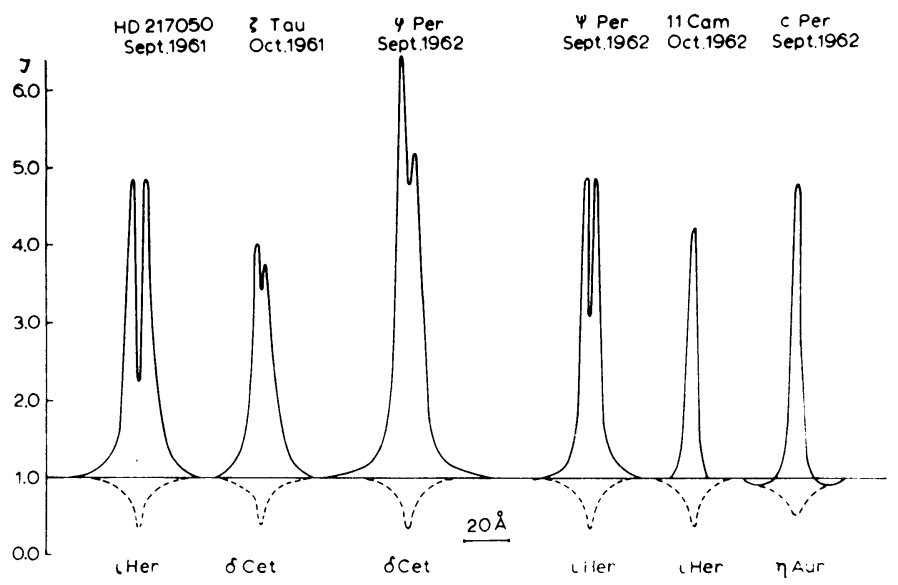

Fig. 1. The profiles of $\mathrm{H} \alpha$ line in the spectra of some Be and B stars (Boyarchuk and Pronik, 1964)

Be stars and shell stars do not form two different groups. The observed differences between $\mathrm{Be}$ and shell spectra is due rather to the dimensions of the envelope and to the angle of inclination of the rotational axis to the line of sight. Figure 4, which is based on a figure given by Hack and Struve (1971), shows schematically the formation of a line in the envelope. If the observer is at $\mathrm{O}_{1}$, the line of sight is parallel to the rotational axis, the profile of the spectral lines will be characterized by stellar absorption on which a central emission due to the extended equatorial parts of the envelope is superimposed. The envelope absorption is absent, because there is no absorbing gas between the stellar surface and the observer. This type of Be star is called 'pole-onstars'. When the observer is at $\mathrm{O}_{3}$, the line of sight is perpendicular to the rotational 


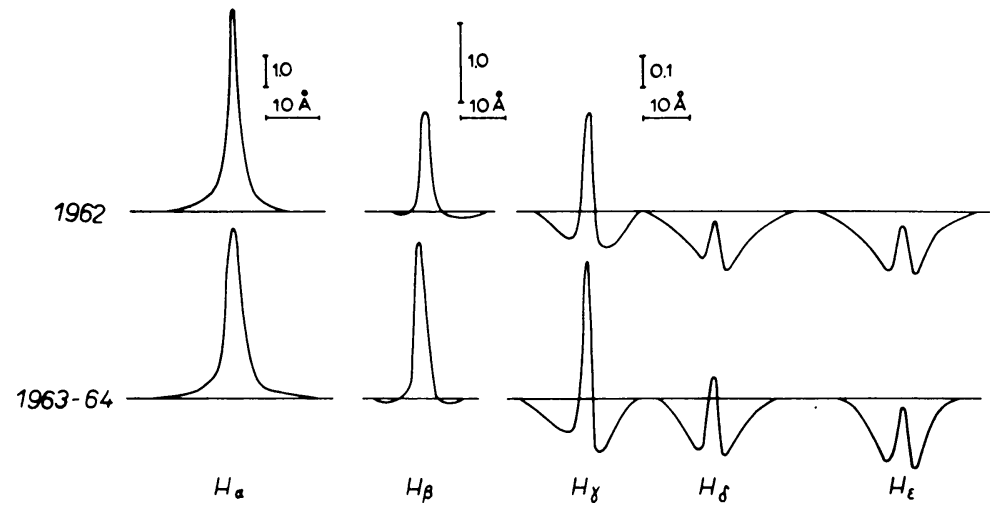

Fig. 2. The profiles of $\mathrm{Ha}$ to $\mathrm{H} \varepsilon$ in the spectra of $\chi$ Oph (Boyarchuk and Pronik, 1965).

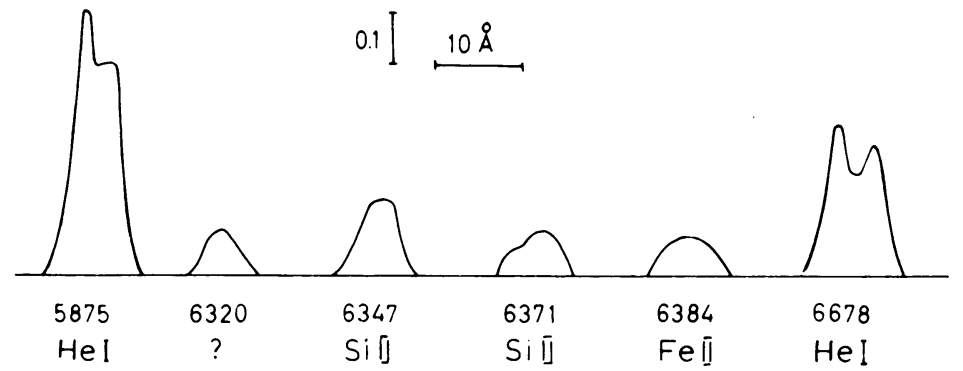

Fig. 3. The profiles of some emission lines in the spectrum of X Per. (Boyarchuk and Pronik, 1965.)

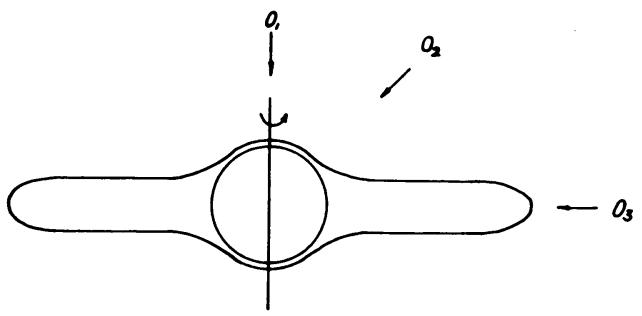

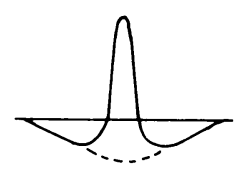

0 ,

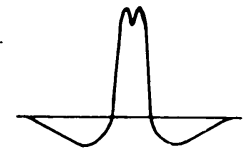

$O_{2}$

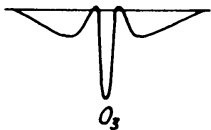

Fig. 4. Sketch of a Be star envelope. 
axis. We observe the stellar absorption lines, which are broadened strongly by stellar rotation. On this line is superimposed the narrow central absorption due to the part of the envelope which is in front of the stellar disk, and two emission components due to the rest of the envelope. The emission components are displaced from the centre by the rotation of the envelope. In this case we have a shell star. If the observer is at $\mathrm{O}_{2}$ we have an intermediate case, which usually is called Be stars.

The most complicated profile belongs to the hydrogen lines which have the strong stellar absorption with Stark wings, two envelope emission components, and central envelope absorption. The simplest profile belongs to the metallic lines, which often are only rather narrow absorption lines. It is easy to understand that the line profile, the ratio of the intensities of the emission components, and of the central absorption depend on the conditions of the excitation and on the gas motion in the envelope. A great amount of data about the displacement of spectral lines formed in the stellar envelope has been accumulated. McLaughlin, Struve, Swings and Merrill were main contributors in this type of work. The best review of results of measurements of line displacements was given by McLaughlin (1961). The main conclusion which can be drawn is that the radial velocities of the central absorption and of the emission components change irregularly in the spectra of most of the stars.

As an example. Figure 5 shows the variation of the radial velocity of emission, $E$, and of absorption, $A$, in the spectrum of the star HD 20336 from 1915 to 1957. Figure 5 shows also the variation of the ratio of the intensities of the blue and of the

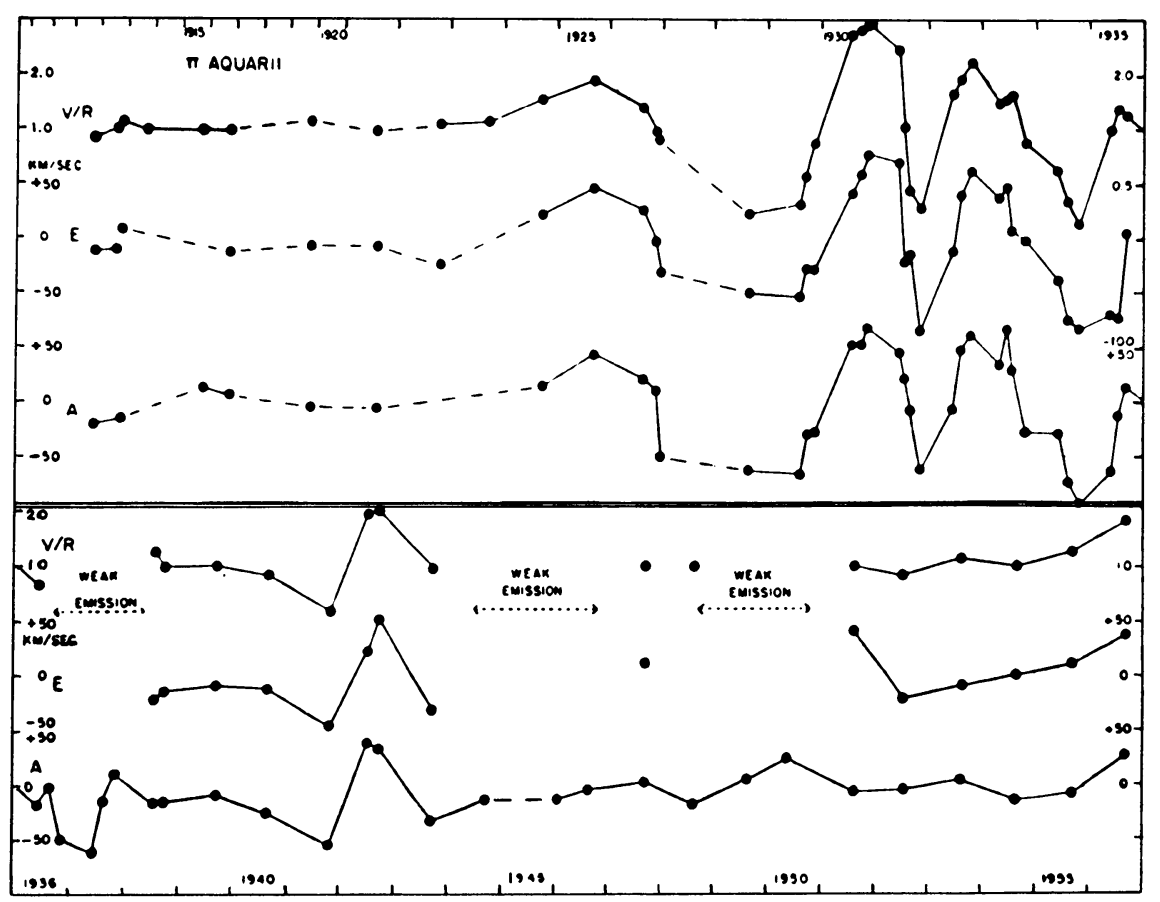

Fig. 5. Velocity and $V / R$ variations of HD 20336 (McLaughlin, 1961). 
red emission components, $V / R$. The quasi-periodic variations were well observed from 1915 to 1935 while from 1940 there are only small non-periodic variations. Most Be stars show similar variations. A few stars show periodic variation of their radial velocities for a long time. For example, $\zeta$ Tau and $\phi$ Per have variations of the radial velocities with periods 132.91 and 126.67 respectively. These variations have been interpreted as due to the orbital motions in binary systems.

Besides the variations of the radial velocities and of the relative intensities of emission components, irregular variations of the total intensity of emission, $E / C$, and of the intensity of the shell spectra have been observed. Sometimes those variations were so large that they lead to the appearance or disappearance of the features of the envelope. McLaughlin (1961) pointed out that sometimes the spectrum of $\pi$ Aqu has no emission. On the other hand, we have the well-known star Pleione. From 1905 on until 1938 it was a normal B5 star with absorption lines greatly broadened by rotation. However, quite suddenly, in 1938, McLaughlin and Mohler discovered that the spectrum of Pleione had hydrogen emission lines together with the shell spectra of ionized metals. Great variations also took place in the spectrum of $\gamma$ Cas.

Thus, the observed radial velocities of envelopes of $\mathrm{Be}$ stars as a rule change irregularly. This means that huge masses of gas move towards and away from the star changing irregularly in velocity and direction. Moreover, as we will see below, the motions involve rather large volumes of matter.

An interesting fact was discovered by Pringle and McNamara (1962). They found that the radial velocities of $\zeta$ Tau determined by absorption lines redward of the Balmer discontinuity are systematically more negative by about $40 \mathrm{~km} \mathrm{~s}^{-1}$ than those determined from lines shortward of the Balmer discontinuity. This fact can be easily explained if the expansional velocity of the envelope decreases outwards. The irregularity of processes which occur in the envelopes of Be stars cause many difficulties for our understanding the reasons of the origin of envelopes.

Let us consider now the character of the local motions of gas. Information about this type of motion can be obtained from line profiles or from the curve of growth (i.e. the ratio between the value of the equivalent width of line and the number of absorption atoms which form it). By analogy with the analysis of spectra of stellar atmospheres we will call the motions found from the line profiles 'macroturbulence' and the motions found from the curve of growth 'microturbulence'.

As far as all Be stars have very large rotational velocities (Sletteback, 1949) the profiles of both the stellar and the envelope lines are broadened by rotation. In such cases, we can use the profiles for the analysis of turbulent motions if the profile width exceeds the rotational width. It was found that the width of $\mathrm{H} \alpha$ emission is about two times larger than that of $\mathrm{H} \beta$ emission in the spectra of shell stars and some other $\mathrm{Be}$ stars (Underhill, 1953; Ringulet-Koswalder, 1963). Underhill (1961) suggested that the motions with the velocities above $1000 \mathrm{~km} \mathrm{~s}^{-1}$ exist in the shell stars. Later Boyarchuk and Pronik (1964) studied the profile of the $\mathrm{H} \alpha$ line in the spectra of some Be stars and shown that the broad wings are due to radiation damping and not to the Doppler effect. Figure 6 shows the analysis of the $\mathrm{H} \alpha$ profile. The observed points 

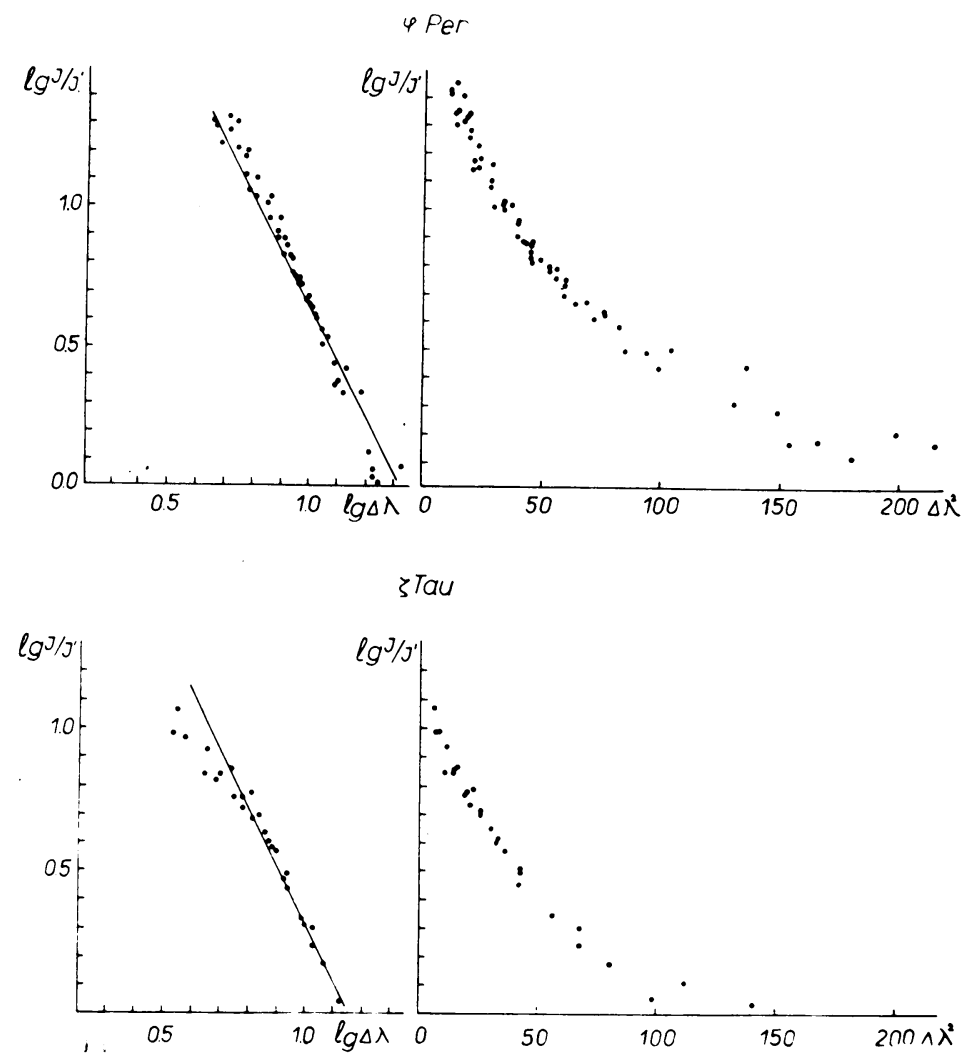

Fig. 6. Analysis of $\mathrm{H} \alpha$ profile.

give the straight line with a slope of about two in the plot of $\lg I$ against $\lg \Delta \lambda$. This slope is the one expected for broadening by radiation damping. There is a significant deviation from a straight line, however, which corresponds to Doppler broadening. Thus the rotation of envelopes leave us a small possibility to use emission line profiles for the analysis of macroturbulence.

The analysis of the equivalent widths gives us another possibility to study motions in the envelopes. In this case we investigate the motions of gas volumes which are smaller than the thickness of the envelope. The value of turbulent velocity is determined by the vertical shift of the observed curve of growth. Therefore this method can be used only for shell stars with strong absorption metallic spectra, which can be observed with a high dispersion. This is the main reason why curves of growth were constructed only for a few stars (Boyarchuk and Pronik, 1963, 1964, 1967; Ozemre, 1967). These investigators found that the value of the turbulent velocity should be changed from $\sim 10 \mathrm{~km} \mathrm{~s}^{-1}$ to $\sim 20 \mathrm{~km} \mathrm{~s}^{-1}$. The value of the turbulent velocity in the envelope of Be stars is larger than the average value found for the atmospheres of supergiants.

The width of emission and absorption lines that arise in envelopes can give some information about the dimensions of the envelope. Struve and Wurm (1938) showed 
that the envelopes of $\mathrm{Be}$ stars are rotating in accordance with the conservation of the angular momentum:

$$
v \cdot r=\text { const }
$$

where $v$ is the rotational velocity at the distance $r$ from the axis of rotation. Struve (1942) was the first to determine the dimensions of the envelope of a Be star. He found that the radius of envelope is equal to about two radii of the star and therefore the dilution factor is about 0.1 . Later investigations confirmed these values. Boyarchuk (1958) found from the study of $12 \mathrm{Be}$ stars that the mean radius of envelope is equal $1.6 R_{\mathrm{star}}$, if the emission lines are used and 2-3 $R_{\mathrm{star}}$, if the absorption lines are used.

Let us consider the conditions of the excitation in the envelope of a Be star. There is a little doubt that the source of the excitation of emission lines in the envelopes is the radiation of a central star. The same mechanism which takes place in the planetary nebulae acts in Be-star envelopes: the stellar radiation ionizes the atoms in an envelope which then undergo recombinations and cascade transitions. The observed emission lines are the result. However, the conditions in the envelopes of Be stars differ significantly from those in planetary nebulae. The envelopes are optically thick for lines of subordinate series. The envelopes have a dilution factor about $10^{-1}-10^{-2}$ while planetary nebulae have a dilution factor much smaller $\sim 10^{-12}$. It is necessary to keep in mind these differences when we calculate the Balmer decrement. First, we have to take into account the ionization from excited levels. Second, we have to consider simultaneously a set of non-linear integral-differential equations. The calculation of Balmer decrements in Be-star envelopes met many mathematical difficulties. There are two main approaches: the moving-envelope theory and the static-atmosphere theory.

These theories may give the two extreme cases with respect to the state of motion inside the envelope. The moving-envelope theory was developed by Sobolev (1946, 1962). He supposed that if the envelope is in a state of motion with varying velocity, photons emitted in the interior region have a probability, $\beta_{i k}$, to leave the envelope without any absorption because of the Doppler effect and to contribute to the formation of emission lines. The introduction of this probability of photons leaving makes it possible to change a system of complicated equations of radiative transfer into a system of rather simple algebraic equations. Many astronomers have calculated the Balmer decrement by using Sobolev's theory.

The most extensive calculations were made by Boyarchuk (1966), by Hirata and Uesugi (1967) and by Ilmas (1971). Boyarchuk gave the Balmer decrement for a wide range of values of the electron temperature, the stellar temperature, the dilution factor and the probability of $L_{\alpha}$-photon exit. The first thirty levels were considered in the solution, a correction being applied for the remaining levels. Ilmas (1971) has taken into account transition by electronic collisions.

The static-atmosphere theory has been developed by Miyamoto $(1949,1952)$ and by Kogure $(1959,1961,1967)$. The envelope is supposed to be static and opaque for the Lyman and Balmer radiations so that the Balmer lines are formed in the outer- 
most part of the envelope. The mathematical difficulties of the solution of the system of transfer equations permit to consider only seven energy levels (discrete + continuum). Figure 7 shows the relation between $\mathrm{H} \gamma / \mathrm{H} \beta$ and $\mathrm{H} \delta / \mathrm{H} \beta$. The observed values were published by Rojas and Herman (1958) for B0-B4 stars (open circles) and for B5-B9 stars (dots) and by Burbridge and Burbridge (1953) for B0-B4 stars (squares). One theoretical curve corresponds to the solution case VII of the static-atmosphere theory (Kogure, 1967), another theoretical curve corresponds to a solution of the moving-envelope theory (Boyarchuk, 1966).

Sobolev's theory gives a good agreement with observations. The static-atmosphere

(e)

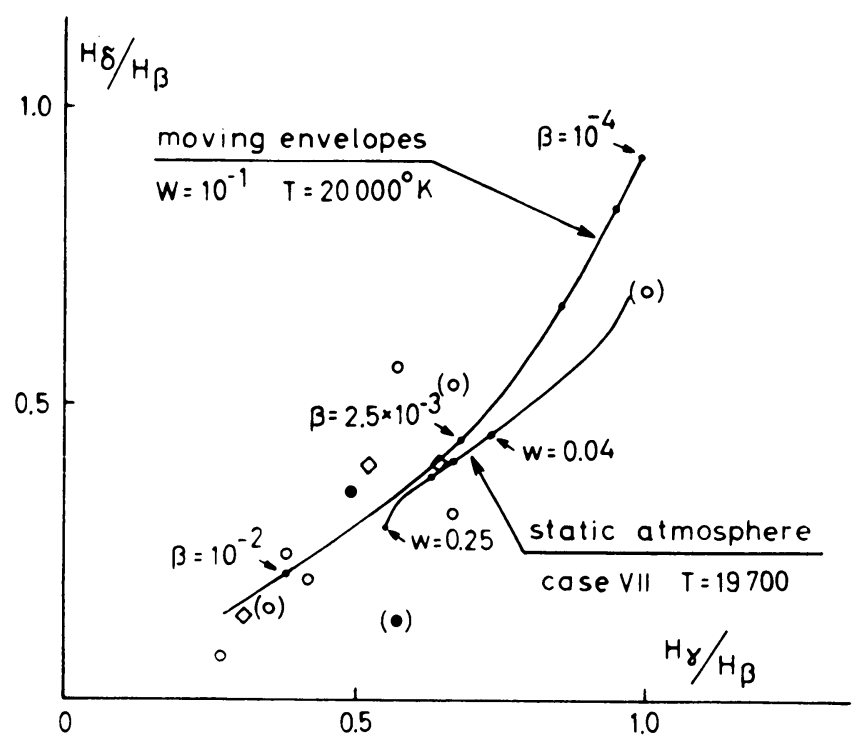

Fig. 7. $(\mathrm{H} \gamma / \mathbf{H} \beta)-(\mathrm{H} \delta / \mathrm{H} \beta)$ diagram.

theory agrees with observations when the dilution factor becomes larger than 0.25 . The latter contradicts the values of $W=0.1,0.01$, which are deduced by the law of conservation of angular momentum.

It should be noted that the observed Balmer decrement depends on the manner of drawing in the underlying background. If an emission line is located above a pure continuum, it is easy to draw a background. But if an emission line is superimposed on strong stellar absorption lines the problem of drawing a background becomes more complicated. The opacity of the envelope for line radiations introduces some additional difficulties in the determination of the intensities of the background stellar radiation. In practice astronomers draw usually the underlying background by an arbitrary interpolation, and this can give a rather large error for weak emission such as $\mathrm{H} \gamma, \mathrm{H} \delta$, etc. 
Several astronomers have investigated emission profiles (Rublev, 1964; Kogure, 1969; Marlborough, 1969). All of them explained a general shape of emission lines, the differences in details due apparently to an inhomogeneity of the envelopes.

Only a few investigations of relative intensities of lines of other elements than hydrogen have been made. It is necessary to point out the calculations in the relative intensities of neutral helium lines by Struve and Würm (1938) and by Wellman (1952). They have shown that the metastable levels are overpopulated under conditions of dilute radiation, and absorption lines arising from levels such as $\lambda 3889$ and $\lambda 3965$ are strengthened. This is in qualitative agreement with observations.

If we have some information about physical conditions in the envelopes, we can apply methods which are used usually in the analysis of the stellar atmospheres. We can use the Inglis-Teller formula

$$
\lg n_{e}=23.26-7.5 \lg n,
$$

(where $n$ is the upper level quantum number of the last Balmer line resolved) for the estimation of the electron density. Boyarchuk and Pronik (1965) have found the value $n_{\mathrm{e}} \sim 2 \times 10^{11} \mathrm{~cm}^{-3}$ for the envelope of $\zeta$ Tau in 1964. Searle (1958), from a study of three shell stars, concluded that the electron densities of these shells are about $5 \times 10^{11} \mathrm{~cm}^{-3}$. We can find values $n_{\mathrm{e}}$ of the same order from tracings published by different astronomers. We conclude that the values $n_{\mathrm{e}}=10^{11}-10^{12} \mathrm{~cm}^{-3}$ are found in large envelopes of Be stars. We cannot estimate the value of $n_{\mathrm{e}}$ for shell envelopes in which spectra the shell lines of envelopes disappear in the first Balmer lines. We can estimate the number of the hydrogen atoms, $N_{2}$, on the second energy level above $1 \mathrm{~cm}^{2}$ of stellar surface by using the approximation of optically thin case (Unsöld, 1939):

$$
W_{\lambda}=\frac{\pi^{2} e^{2} \lambda^{2}}{m c^{2}} f N_{e},
$$

where $W_{\lambda}$ is the equivalent width of the shell absorption lines and $f$ is the oscillator strength.

Boyarchuk (1958) has studied ten Be stars. He found that the values of $\lg \mathrm{N}_{2}$ vary from about 16 for $\zeta$ Tau to 13 for 23 Tau. It is easy to calculate that such strong envelopes as the envelope of $\zeta$ Tau are opaque to the centers of the Balmer lines, but are transparent beyond the Balmer limit. The optical thicknesses of envelopes beyond the Lyman limit of all but $\zeta$ Tau stars are inferred to be $\tau_{L} \leqslant 1$. The envelope of $\zeta$ Tau has the value of $\tau_{L} \sim 10$. It means that the stellar radiation cannot ionize atoms in the envelope which have ionization potential higher than $13.6 \mathrm{eV}$. As a result, we observe in the spectrum of $\zeta$ Tau the numerous lines of the one-ionized metals. The shell metallic lines are not observed in the spectra of envelopes for which the value of $\lg N_{2}<15$. The problem of the origin of the envelope is still unsolved. It is known that $\mathrm{Be}$ stars are rotating very rapidly. Struve was the first to propose that the envelopes of Be stars formed through rotationally forced ejection. These stars are unstable at their equators and are losing mass through rotational break-up. But recently 
Slettebak (1966) has found that the largest observed rotational velocities for stars O9.5-F0 V are always below the computed equatorial break-up. If a stable continuous ejection of gas caused by the stellar rotation takes place from stellar surface we should observe the stable expansion of the gas in the envelope. As we have seen earlier, on the average the envelopes do not expand. Thus, stellar rotation is not the main reason for the formation of envelopes; it creates favourable conditions for gas ejection, but an additional reason is needed. That reason, that mechanism does not have to work continually: its power changes with time.

Boyarchuk (1959) has proposed that forces similar to those producing solar activity act on the surface of Be stars. Limber and Marlborough (1968) and Henriksen (1969) assume that magnetic fields play an important role. There is a small group of hot stars which resemble Be stars in some respects. This is the group of $\mathrm{P}$ Cygni stars. The main features of the spectra of P Cygni stars are the emission lines accompanied by violetshifted absorptions. But in the case of a P Cyngi star we have an extended atmosphere rather than an expanding envelope and these stars will be discussed in other reports.

\section{Envelopes of the Wolf-Rayet Stars}

Wolf-Rayet stars are characterized by the presence in their spectra of numerous broad emission lines. Their spectra also contain a few absorption lines which arise from metastable energy levels, overpopulated because of dilution effects, and from relatively low levels.

Wolf-Rayet stars may be separated into two groups. The first group, indicated as WC, shows emission lines of carbon and oxygen, and the second group, WN, shows emission lines of nitrogen. Underhill (1958) suggested that the difference is due to a higher level of excitation in WN envelopes than in WC envelopes. But Kuhi (1968b) doubts this explanation, because $\mathrm{N}$ III and $\mathrm{C}$ III have similar ionization potentials.

The main process of excitation is ionization of the gas by short-wave stellar radiation and recombination. Several lines of $\mathrm{He}$ II, $\mathrm{O}$ III and $\mathrm{N}$ III are excited by monochromatic processes of Bowen's type.

Some authors have determined the temperature of the excitation radiation by Zanstra's method. Beals (1940) gives temperatures ranging from $59000 \mathrm{~K}$ to 110000 $\mathrm{K}$ for seven stars. Higher temperatures are found when lines from ions of low ionization potential are used. It can be interpreted that the level of ionization and excitation decreases outward quite significantly in :Wolf-Rayet envelopes. Further investigations (Aller, 1943; Voronzov-Veliaminov, 1948; Aller and Faulkner, 1964) confirm Beals' results. However, it should be noted that conditions in envelopes of WR stars differ significantly from those of planetary nebula and Zanstra's method may lead to erroneous conclusions. Miyamoto (1952) points out that conditions in Wolf-Rayet envelopes probably resemble those in Be envelopes. He finds by using his own theory that the temperatures range between $30000 \mathrm{~K}$ and $41000 \mathrm{~K}$ - lower than those suggested by Beals. Rublev (1964) has determined the electron temperatures in envelopes 
of two Wolf-Rayet stars. He studied Pickering series of He II and found that the electron temperature was about $25000 \mathrm{~K}$.

Underhill (1968) points out that the fact that no forbidden lines are observed in any spectra of WR stars indicates that their envelopes are not very rarefied, $n_{\mathrm{e}} \gtrsim 10^{10}$ $\mathrm{cm}^{-3}$. On the other hand $n_{\mathrm{e}} \lesssim 10^{14} \mathrm{~cm}^{-3}$ because no significant Stark broadening of the $\mathrm{He}$ II lines is observed. Wallerstein (1968) estimates $n_{\mathrm{e}} \sim 10^{11}$ from the X-ray intensity of a source which he relates to the WR star HD 211853.

Now let us consider motions in the envelopes of WR stars. The profiles of the emission lines give us important information. The emission lines which are observed in the spectra of WR stars are of two types: those with a gaussian profile (He II $\lambda$ 4686) and those with a broad flat top (He I $\lambda$ 5876). The profiles of the lines in the first group correspond to broadening by turbulent motions with velocities of the order of $500 \mathrm{~km} \mathrm{~s}^{-1}$ to $1000 \mathrm{~km} \mathrm{~s}^{-1}$. The second group of profiles is characteristic of rapidly expanding spherical envelopes. The velocities of expansion are about $1000 \mathrm{~km} \mathrm{~s}^{-1}$. (Beals, 1931; Sobolev, 1947).

The expansion of envelopes is confirmed by the presence of blue-shifted absorptions. The data of the measurements given by Hack and Struve (1971) show that the average of velocities of expansion is about $1200 \mathrm{~km} \mathrm{~s}^{-1}$. The velocities are a little bigger for WC stars than for WN stars and in both cases they are larger in stars of lower temperatures. The observed velocities of expansion exceed the escape velocity $\left(V_{\text {esc }} \sim 600\right.$ $\mathrm{km} \mathrm{s}^{-1}$ for almost all stars.

Many WR stars are double stars. This makes it possible to determine the dimensions of the envelopes of WR stars, as has been done by Hiltner (1949), Kron and Gordon (1950) and Munch (1950). The most detailed investigation was made by Kuhi (1968). He observed several eclipses of the WR component of V 444 Cyg with a photoelectric spectrum scanner. He obtained a variety of shapes of eclipse curves for different lines. From the depth of the minimum we can compute the radius of the occulated WR envelope, assuming that the disk of the envelope has uniform surface brightness. Kuhi finds that the $\mathrm{He}$ II envelope has a radius of $16.9 R_{\odot}$, while the $\mathrm{N} v$ envelope has a radius of $31 R_{\odot}$. The WR component has a radius of $2 R_{\odot}$ (Wilson, 1942). This means that the dilution coefficient in envelopes of WR stars is about $10^{-2}-10^{-3}$, i.e. ten times smaller than in the envelopes of Be stars. Since lines of higher ionized ions have smaller width, the gas in envelopes of WR star must be radially accelerated. Kuhi points out also that there are lines whose intensities vary in a strange manner. They do not show any minimum or show a minimum early, before the minimum of continuous radiation. Kuhi explained these facts by inhomogeneity in the envelope caused by gas streams in the binary system. Kuhi's observations confirm the existence of a stratification of physical conditions in envelopes of WR stars. In general, the lines of higher ionized ions arise in deeper level than that of lower ionized ions.

The first model WR star was proposed by Beals (1930). He suggests that the broad emission lines should be produced in a radially expanding envelope surrounding the star. This model explains some features of WR stars, but there are two objections at least. First, not all emission lines have flat topped profiles accompanied by violet-dis- 
placed absorption components. Second, Wilson (1940) pointed out the absence of the expected phase shift between photometric and spectroscopic times of minima. In order to eliminate these difficulties, Underhill (1966) proposed more complicated model:

WR star has a moderately extended, moderately dense atmosphere in chaotic motion above a compact photosphere. This atmosphere is probably equivalent to the luminous disk of Kron and Gordon (1950). We must assume that the star is surrounded by a low density, rapidly expanding atmosphere (envelope) which is significantly opaque chiefly in lines which are strengthened under conditions of high temperature and moderate dilution.

It should be noted that although many papers have been published about WR stars, we do not know for sure the physical conditions $\left(T_{\mathrm{e}}, n_{\mathrm{e}}\right)$ in their envelopes. We do not know what is the mechanism of formation of envelopes or what kind of forces work in the envelopes.

The Be stars and WR stars are groups of stars in which envelopes have been studied rather in detail. There are other objects that may have envelopes. But those envelopes have been studied only roughly. In some cases, we do not know whether we observe envelope or another phenomenon. Below we will consider several examples:

\section{U Geminorum Stars and Old Novae}

These stars are close binaries. The main component is surrounded by an envelope, which has the shape of a disk (Prendergast, 1960). The envelopes rotate with velocities of about $600 \mathrm{~km} \mathrm{~s}^{-1}$ (Krzeminski, 1965). The envelopes have the electron density of about $10^{13} \mathrm{~cm}^{-3}$ and the radius is about $10^{10} \mathrm{~cm}$ (Gorbatskij, 1970).

\section{Symbiotic Stars}

The term 'Symbiotic Stars' designates those astronomical objects whose spectra represent a combination of absorption features of a low temperature star with emission lines of high excitation. Swings and Struve (1941) have suggested that symbiotic stars are binaries: one of the components is a late-type giant, and the other a hot, small star which is the source of the excitation of an envelope surrounding both components. Boyarchuk (1970) has determined the size of an envelope $\sim 10^{4} R_{\odot}$ and the electron density $\sim 10^{7} \mathrm{~cm}^{-3}$. Thus the envelopes in which the emission lines originate are probably more closely related to planetary nebulae than to true stellar envelopes.

\section{T Tauri Stars}

This is a group of irregular variables of spectral class $G$ which have emission lines in their spectra. Their peculiar spectral characteristics and other properties have been reviewed by Herbig (1962). The strongest emission lines (hydrogen and Ca II) often have shortward-displaced absorption component. Herbig has suggested also that the forbidden lines observed in the spectra of most $\mathrm{T}$ Tauri stars arise in a circumstellar 
envelope. Kuhi (1964) has interpreted the broad emission lines of $\mathrm{H}$ and $\mathrm{Ca}$ II in terms of an expanding envelope. A fit of computed line profiles to observed ones then gives the density at the surface of the star as $\sim 10^{10}$ atom $\mathrm{cm}^{-3}$ and expansion velocities of $\sim 150 \mathrm{~km} \mathrm{~s}^{-1}$ as the average values for six stars. But some important characteristics of T Tauri stars are unknown still. For instance, we do not know what is the excitation source for the emission. It is possible that here we have a mechanism like that in the solar chromosphere. The T Tauri phenomenon is more closely related to stellar atmospheres than to envelopes. Recently, Walker (1969) has drawn attention to the fact that many $\mathrm{T}$ Tauri stars with the strong ultra-violet excess have redward-displaced absorption components of hydrogen and $\mathrm{Ca}$ II emission lines. These stars have an inverse P Cyg spectrum. Walker proposed that the inverse P Cyg spectrum in the T Tauri stars with ultra-violet excesses indicates actual infall of material. Thus I am not sure that in the case of $\mathrm{T}$ Tauri stars we are concerned with true envelopes. It seems to me the $\mathrm{T}$ Tauri phenomenon is more complicated.

In conclusion, it should be stressed that all of the problems of envelopes which were formulated by Otto Struve thirty years ago still require answers.

\section{References}

Aller, L. H.: 1943, Astrophys. J. 97, 135.

Aller, L. H. and Faulkner, D. I.: 1964, Astrophys. J. 140, 167.

Beals, C. S.: 1930, Publ. Dominion Astrophys. Obs. 4, 228.

Beals, C. S.: 1931, Monthly Notices Roy. Astron. Soc. 91, 966.

Beals, C. S.: 1940, J. Roy. Astron. Soc. Can. 34, 169.

Boyarchuk, A. A.: 1958, Mem. Soc. Roy. Sci. Liège, 4ème Série 20, 159.

Boyarchuk, A. A.: 1960, Voprosi kosmogonyi 7, 231

Boyarchuk, A. A.: 1966, Izv. Krymsk. Astrofiz. Obs. 35, 45.

Boyarchuk, A. A.: 1970, in A. A. Boyarchuk and R. E. Gershberg (eds.), Eruptivnye Zvezdy, Nauka, Moscow, p. 148.

Boyarchuk, A. A. and Pronik, I. I.: 1963, Izv. Krymsk. Astrofiz. Obs. 29, 268.

Boyarchuk, A. A. and Pronik, I. I.: 1964, Izv. Krymsk. Astrofiz. Obs. 31, 3.

Boyarchuk, A. A. and Pronik, I. I.: 1965, Izv. Krymsk. Astrofiz. Obs. 33, 195.

Boyarchuk, A. A. and Pronik, I. I.: 1967, Izv. Krymsk. Astrofiz. Obs. 36, 203.

Burbidge, G. R. and Burbidge E. M.: 1953, Astrophys. J. 118, 252.

Gorbatsky, V. G.: 1970, in A. A. Boyarchuck and R. E. Gershberg (eds.), Eruptivnye Zvezdy, Nauka, Moscow, p. 63.

Hack, M. and Struve, O.: 1971, Stellar Spectroscopy 2, Trieste, p. 14.

Henriksen, R. N.: 1969, Astron. Astrophys. 1, 457.

Herbig, G. H.: 1962, Adv. Astron. Astrophys. 1, 47.

Hiltner, W. A.: 1949, Astrophys. J. 110, 95.

Hirata, R. and Uesugi, A.: 1967, Contr. Kwasan Obs. Kyoto No. 156.

Ilmas, M.: 1971, in The Emission Lines in the Stellar Spectra, Tartu, p. 47.

Kogure, T.: 1959, Publ. Astron. Soc. Japan 11, 127, 278.

Kogure, T.: 1961, Publ. Astron. Soc. Japan 13, 335.

Kogure, T.: 1967, Publ. Astron. Soc. Japan 19, 30.

Kogure, T.: 1969, Astron. Astrophys. 1, 253.

Kron, G. E. and Gordon, K. C.: 1950, Astrophys. J. 111, 454.

Krzeminski, W.: 1965, Astrophys. J. 142, 1051.

Kuhi, L. V.: 1964, Astrophys. J. 140, 1409.

Kuhi, L. V.: 1968a, Astrophys. J. 152, 101.

Kuhi, L. V.: 1968b, in K. B. Gebbie and R. N. Thomas (eds.), Wolf-Rayet Stars, Washington, p. 108. 
Limber, D. N. and Marlborough, J. M.: 1968, Astrophys. J. 152, 181.

McLaughlin, D. B.: 1961, J. Roy. Astron. Soc. Can. 55, 76.

Marlborough, J. M.: 1969, Astrophys. J. 156, 135.

Miyamoto, S.: 1949, Jap. J. Astron. 1, 17.

Miyamoto, S.: 1952, Publ. Astron. Soc. Japan 4, 1.

Münch, G.: 1950, Astrophys. J. 112, 266.

Ozemre, K.: 1967, Ann. Astrophys. 30, 495.

Prendergast, K.: 1960, Astrophys. J. 132, 162.

Pringle, J. K. and McNamara, D. M.: 1962, Publ. Astron. Soc. Pacific 74, 525.

Ringuelet-Kaswalder, A. E.: 1963, Publ. Astron. Soc. Pacific 75, 323.

Rojas, H. and Herman, R.: 1958, Mem. Soc. Roy. Sci. Liège, 4ème Série 20, 198.

Rublev, S. V.: 1964, Astron. Zh. 41, 63 (Soviet Astron. 8, 45).

Searle, L.: 1958, Astrophys. J. 128, 61.

Slettebak, A.: 1949, Astrophys. J. 110, 498.

Slettebak, A.: 1966, Astrophys. J. 145, 126.

Sobolev, V. V.: 1947, Moving Stellar Envelopes, Leningrad.

Sobolev, V. V.: 1962, Astron. Zh. 39, 632 (Soviet Astron. 6, 531).

Struve, O.: 1942, Astrophys. J. 95, 134.

Struve, O. and Würm, K.: 1938, Astrophys. J. 88, 84.

Swings, P. and Struve, O.: 1941, Astrophys. J. 93, 356.

Underhill, A. .: 1953, Monthly Notices Roy. Astron. Soc. 113, 477.

Underhill, A. B.: 1958, Mem. Soc. Roy. Sci. Liège, 4ème Série 20, 17.

Underhill, A. B.: 1961, Publ. Dominion Astrophys. Obs. 11, 405.

Underhill, A. B.: 1968, in K. B. Gebbie and R. N. Thomas (eds.), Wolf-Rayet Stars, Washington, p. 195.

Unsöld, A.: 1938, Physik der Sternatmosphären, Springer Verlag, Berlin, p. 290.

Vorontsov-Velyaminov, B. A.: 1958, Mem. Soc. Roy. Sci. Liège, 4ème Série 20, 55.

Walker, M.: 1969, in L. Detre (ed.), Non-Periodic Phenomena in Variable Stars, Academic Press, New York, p. 103.

Wallerstein, G.: 1968, Astrophys. J. Letters 151, L121.

Wellman, P.: 1952, Z. Astrophys. 30, 80.

Wilson, O. C.: 1940, Astrophys. J. 91, 3, 79.

Wilson, O. C.: 1942, Astrophys. J. 95, 402. 